\title{
The Field Inspection and Analysis on Electric Energy Metering Device
}

\author{
Jia Qiuxia, Zhao Yan, Wang Tianhao, Wang Chuang, Shi Xiao, Hu Yang, Su Yang, \\ Tian Zhili, Liu Xiao, Hao Wenfeng \\ Fushun Power Supply Company, Liaoning Electric Power Company Limited, State Grid, China
}

Keywords: electric energy metering device; on-site inspection; the change pass; variable measuring box; watt-hour meter; transformer

\begin{abstract}
In the process of rapid social development, there is an increasing demand for electricity in all walks of life, it makes people pay more and more attention to the accuracy of power measurement. In order to ensure that legitimate rights and interests of both sides are effectively guaranteed, it is necessary for on-site inspection. And electric energy metering device improves the accuracy and reliability of the electric energy metering, accurate trade information is made for power supply. Moreover, it is of positive significance to improve the quality of electricity monitoring and to improve the quality of service quality of electric power enterprises by strengthening the on-site inspection of electric energy metering devices.
\end{abstract}

\section{Introduction}

The current supply and electricity settlement is based on the data measured by the metering device. Once the measuring equipment is inaccurate, it will affect the legitimate interests of the two parties. This also makes the power supply both sides to the measurement device field inspection work is very concerned. By strengthening the variable threshold, metering box, changeable watt-hour meter, transformer and metering device, the precision of measurement is effectively improved, not only the legitimate rights and interests of the power supply are effectively guaranteed both sides, but also fair and lawful operation of the electric power enterprise has very important significance.

\section{The Metering Device Inspection of the Changing Pass on-site}

\subsection{Preparation before Testing}

Before the test starts, the measuring device needs to be in the working state, the device is required to be in the constant load condition when conducting the metering device of transformer on-site inspection. If there is not normal open state, portable generator can be made to generate virtual load for replacement, but since it has certain limitation, so changes in the transformer, secondary wiring, and error judgment of watt-hour meter can only under special conditions.

\subsection{Detection Error}

At the scene of the specific detection, often the integrated test method is used, from the variable current transformer to the inspection instrument on-site, the voltage and current can be done through the use of current clamp, but just for a power measurement at this time. The tester still needs to be used to a power conversion into a second, it also makes watt-hour meter, transformer and the secondary wiring error constitute the watt- hour meter, error is over after the inspection on-site is passed.

\subsubsection{Processing Methods under Unqualified Test Results}

When detection error is not qualified, the secondary wiring, watt-hour meter, and phase sequence of transformer need to be checked. Through the voltage and current samples, the error of watt-hour meter, watt-hour meter and secondary wiring whether qualified can make use of vector diagram of 
impedance tester instrument to test.

\subsubsection{Application Analysis}

The field test of the metering device is not only fast but also keeps the metering device in a qualified state. In the actual inspection, the appearance of the metering device should be checked first, then the error is combined with the error. If the test is qualified, the inspection of device on-site is also qualified. The last error processing, testing two flash wiring and watt- hour meter, transformers, priority detection instruments of malfunction, the final inspection failure probability of small appliances, so fault source can be the fastest to determine, and the cause of the problem is found out in time.

\subsection{Field Test of the Distribution Transformer}

\subsubsection{Test Appearance}

The appearance detection and the high - arch and low - gauge method are the same for the field inspection of the transformer.

\subsubsection{Compiling Relevant Vector Diagram by Combining the Error of the Electric Energy Meter}

In the process of detection, the testing requirements need to be consistent with field testing apparatus, often change with the load chosen as test object. Because high side metering box is a changeable terminal, and the second head end in a device, this does not lead to the appearance to match variable metering box secondary wiring head end for inspection. Wrong line or even transformer in the watt-hour meter fails, it also can carry on the accurate measurement of electricity, so it is not appropriate to match changed from watt-hour meter, error metering box system error is difficult to judge. As the high arch height device, it is necessary to select the appropriate test sense in the inspection process, and the tester is used to generate the metering graph and various parameters, so as to check whether the secondary wiring is correct. When the error condition of vector diagram exists, it is necessary to find out the error line and calculate the coefficient $\mathrm{K}$.

\subsubsection{Comparing the Power and Verifying the Results}

Two testers can be used to set the different power, to make a correction coefficient $\mathrm{K}$ in counting, with close to power ratio, the wrong wiring and correct rate are found out effectively, and the correctness of the result is preliminary verified.

\section{Application Analysis}

In the process of testing metering box, high voltage transformer cannot test any times, but this kind of transformer is usually used to measure electricity, and the percent of pass is judged by comprehensive error, but the all testing error of the site is not reality. So in actual work, instrument often is used to measure the low voltage power, using high and low voltage comparison method to decide on the operation of the metering device, when the correction coefficient $\mathrm{K}$ is equal to the time, indicating that the operation of the metering box is normal.

\section{The Field Inspection of Electricity Meters}

When the current electricity meter is field tested, it will be tested by photoelectric sampling or electric signal sent. Watt-hour meter tester is chosen to measure voltage, current phase, and it owns discriminant function, the site conditions and inspection requirements are ensured. Tested watt-hour meter is in the actual load condition, in order to test the error of watt- hour meter. In the process of concrete testing, watt-hour meter calibration cycle is checked, the record is checked, the loss pressure of the battery usage, watt-hour meter, watt- hour meter and metering transformer between the secondary wiring, anomalies are found to handle in a timely manner. The measurement errors and unreasonable measurement are checked. 


\subsection{Big Test of Transformer Load}

Field tests found that a large part of the transformer secondary load is greater than the actual load, it is the operation of a transformer under light load condition and out-of-tolerance condition appear easily in the process and running. In the current new substation and electric field of the construction, the transformer secondary load is lighter, microcomputer protection and some advanced technologies are continuous used in transformer protection, it also reduces the secondary load. And the current voltage transformer has a large secondary section, which will further reduce the secondary load. However, in the transformer design of the secondary load, it is necessary to take a comprehensive consideration on the location of the transformer and the combination of the length, cross section and load characteristics of the secondary wires. In test, not just in the rated load condition down to test the transformer load, to in the process of detection of actual load and rated load difference size to focus on, once large difference between them is found, the actual testing is simulated, in order to determine whether there is a super bad phenomenon.

\subsection{Pressure Drop Test of Voltage Transformer}

In the comprehensive error of electric energy measurement, the error caused by voltage transformer is often the largest. The error of the voltage drop in the secondary circuit of the transformer is the dynamic error, and it has the leading effect on the error of the metering device. The increase of resistance between fuse and block is an unstable factor affecting the voltage drop. And over time, the voltage fuse resistance will increase. In addition, the contact resistance of wire thickness and switching relay, terminal row and conductor resistance will affect the voltage drop of the transformer circuit, thus affecting the error of the measuring device. For $35 \mathrm{kV}$ and above transformer circuit voltage drop, at least two years for one test. pressure drop tester is selected to test in the transformer and is forbidden to have a secondary short circuit. It is wrong to use the test instrument to check the phase sequence of the transformer. The side wiring of the transformer should be connected to the first terminal of the second lead wire, and the power meter side wiring shall be connected to the box cover. the voltage side is connected firstly then the power meter side.

\section{Conclusions}

In the testing process of electric energy measurement device, there are many uncertain circumstances, so the need for specific types of metering device adopts error test method or power ratio method, in order to improve the efficiency of detection, improve the detection accuracy.lt is helpful to improve the reliability of electric energy measurement by strengthening the field detection of electric energy metering device. Although the field current will be restricted to many factors in the process of electric energy metering device for testing, as the continuous improvement of detection technology, the electric energy metering device testing will improve quality, the accuracy of the power supply the electricity measurement is ensured better to promote the healthy and sustainable development of the electric power enterprise.

\section{References}

[1] Xie Lubin, Luo Xiuhong, Hu Xinmei. Discussion on the on-site inspection cycle of the electric power metering device of the special transformer [J], Electric switch, 2013 (1).

[2] Cheng Yingying, Yang Huaxiao, et al. Study on operation error analysis and state evaluation method of electric power metering devices [J]. New technology of electrical energy, 2014 (5).

[3] Shao Yanying. Several problems in the installation and operation of electric power metering devices [J]. Technology and enterprises, 2013 (22).

[4] Zhai Lei, Li Yuzhu. Analysis and processing of on-site problems of electric energy metering devices [A]. The 12th excellent paper compilation of shandong electrical engineering institute [C], 2011. 
[5] Bao Weidong, Liu Bing. Field calibration method of electric energy metering device [J]. Electrical measurement and instrumentation, 2006.

[6] Ruan Zhijun. Field calibration research and error analysis of electric energy metering devices. Enterprise technology development, 2012. 